\title{
Total Widths And Slopes From Complex Regge Trajectories
}

\author{
S. Filipponi ${ }^{\mathrm{a}, 1}$, G. Pancheri ${ }^{\mathrm{b}, 2}$ and Y. Srivastava ${ }^{\mathrm{a}, \mathrm{c}, 3}$ \\ ${ }^{a}$ Physics Department-University of Perugia and INFN-Perugia \\ via Pascoli, I-06100 Perugia, Italy. \\ ${ }^{\mathrm{b}}$ Laboratori Nazionali di Frascati dell' INFN \\ Frascati, Rome, Italy. \\ ${ }^{\mathrm{c}}$ Physics Department-Northeastern University \\ Boston, Mass, USA.
}

\begin{abstract}
Maximally complex Regge trajectories are introduced for which both $\operatorname{Re} \alpha(s)$ and $\operatorname{Im} \alpha(s)$ grow as $s^{1-\epsilon}$ ( $\epsilon$ small and positive). Our expression reduces to the standard real linear form as the imaginary part (proportional to $\epsilon$ ) goes to zero. A scaling formula for the total widths emerges: $\Gamma_{T O T} / M \rightarrow$ constant for large $\mathrm{M}$, in very good agreement with data for mesons and baryons. The unitarity corrections also enhance the space-like slopes from their time-like values, thereby resolving an old problem with the $\rho$ trajectory in $\pi N$ charge exchange. Finally, the unitarily enhanced intercept, $\alpha_{\rho} \approx 0.525$, is in good accord with the Donnachie-Landshoff total cross section analysis.
\end{abstract}

\section{Introduction}

It is well known that hadronic mass spectra can be described quite reasonably through real, linearly rising Regge trajectories. Veneziano model and hadronic string models are anchored on such a hypothesis. On the other hand, unitarity and analyticity require the addition of an imaginary part thereby providing total widths for the resonances as well as giving some curvature to the trajectories. The modest aim of our work is as follows. Using the positivity of the imaginary part and the positivity of the slope, it is observed that the real parts

\footnotetext{
1 Silvana@pg.infn.it

2 Pancheri@lnf.infn.it

3 Srivastava@pg.infn.it
}

Preprint submitted to Elsevier Preprint 10 October 2018 
of trajectories can rise no faster than linearly. We then introduce the notion of maximally complex trajectories for which both the real and the imaginary parts grow in the same way, i.e.,

$$
\left.\begin{array}{l}
\operatorname{Re} \alpha(s) \\
\operatorname{Im} \alpha(s)
\end{array}\right\} \longrightarrow s^{1-\epsilon}\left\{\begin{array}{l}
\cos \pi \epsilon \\
\sin \pi \epsilon
\end{array} \quad \text { for } s \rightarrow \infty\right.
$$

where $\epsilon$ is small but positive. Physically, our hypothesis embodies the dictum that "strong interactions are as strong as they can be". It is also satisfactory mathematically since a small imaginary part can only curve $|\alpha(s)|$ downwards from linearity. The limit $\epsilon$ equals zero corresponds to no imaginary part and

a linear real trajectory. Implications of the resulting deviations from linearity are studied for the real parts to discover significant changes in the intercept, in agreement with experimental observations. On the other hand, analysis of the imaginary parts leads us to a pleasing result that total widths of high mass resonances grow linearly with mass, a fact which we show to be true experimentally, [1], both for mesons and for baryons.

\section{Formalism}

Under the assumption that the complex pole function $\alpha(s)$ has:

(1) • a single zero at $s=-s_{0}$

- with a positive derivative;

(2) a unitarity branch cut for $s \geq \Lambda^{2}$, with $\operatorname{Im} \alpha(s) \geq 0$;

it is possible to show that its asymptotic behavior is bounded as follows

$$
\left|\frac{1}{s}\right| \leq|\alpha(s)| \leq|s| \quad \text { for }|s| \rightarrow \infty
$$

To show that $|\alpha(s)| \leq|s|$, let us assume the contrary, i.e., that $|\alpha(s)| \rightarrow|s|^{1+\delta}$, $(\delta>0)$, as $|s| \rightarrow \infty$. Now we write a dispersion relation for the inverse function $1 / \alpha(s)$, which has a single pole at $s=-s_{0}$ (with a positive residue)

$$
\frac{1}{\alpha(s)}=\frac{A}{s+s_{0}}-\int_{\Lambda^{2}}^{\infty} \frac{d s^{\prime}}{\pi} \frac{\operatorname{Im} \alpha\left(s^{\prime}\right)}{\left(s^{\prime}-s-i 0^{+}\right)\left|\alpha\left(s^{\prime}\right)\right|^{2}}
$$


Taking the asymptotic limit of the previous Eq.(3), one has

$$
\frac{1}{|\alpha(s)|} \longrightarrow \frac{1}{|s|}\left[A+\int_{\Lambda^{2}}^{\infty} \frac{d s^{\prime}}{\pi} \frac{\operatorname{Im} \alpha\left(s^{\prime}\right)}{\left|\alpha\left(s^{\prime}\right)\right|^{2}}\right]
$$

Since, $A$ is positive and the integral in Eq.(4) is positive and convergent, $\frac{\operatorname{Im} \alpha}{|\alpha|^{2}}<\frac{1}{s^{1+\delta}}$, the sum can not vanish. Hence, we have proof ad absurdum. A similar derivation holds also for the lower bound condition: $|\alpha(s)| \geq|1 / s|^{1+\delta}$

Our hypothesis of maximum complexity may be written in the following suggestive "renormalized" form

$$
\alpha(s) \rightarrow \bar{\alpha}(s) Z(s ; \epsilon)
$$

where, $\bar{\alpha}(s)$ symbolizes the real, linear "unrenormalized" trajectory and $Z(s ; \epsilon)$ is the complex "renormalization" factor with the boundary conditions

$$
\begin{aligned}
& Z(s ; \epsilon=0)=1 \\
& |Z(s ; \epsilon)| \rightarrow|s|^{-\epsilon} \quad \text { as }|s| \rightarrow \infty
\end{aligned}
$$

The above Eq.(6) makes both Re and $\operatorname{Im} \alpha(s)$ to grow like $s^{1-\epsilon}$, and the asymptotic phase of $\alpha$ goes to a constant. This leads total widths to grow like $\sqrt{s}$ as shown below.

Writing the contribution of a trajectory to an angular momentum $J$ partial wave amplitude as

$$
T(J, s)=\frac{\beta(s)}{J-\alpha(s)}
$$

and comparing it to a Breit-Wigner form for a given $J$ resonance, it is easily seen that for a resonance of mass $M$ and total width $\Gamma_{T O T}(M)$

$$
\operatorname{Im} \alpha\left(M^{2}\right) \approx \operatorname{Re} \alpha^{\prime}\left(M^{2}\right) M \Gamma_{T O T}(M)
$$

Since for small $\epsilon, \operatorname{Im} \alpha(s)$ is proportional to $\epsilon$ and grows for large $s$ as $s^{1-\epsilon}$, we have the approximate result

$$
\frac{\Gamma_{T O T}(M)}{M} \longrightarrow \tan (\pi \epsilon) \quad \text { for large } M
$$




\section{A Model for Maximally Complex Regge Trajectories}

Let us write a dispersion relation for $\eta(s)=\log \frac{\alpha(s)}{s+s_{0}}$, normalize $\alpha(s)$ at a threshold called $\Lambda$ and impose that it has a zero at $-s_{0}$ :

$$
\alpha(s)=\left(\frac{s+s_{0}}{\Lambda^{2}+s_{0}}\right) \alpha\left(\Lambda^{2}\right) e^{\left[\left(s-\Lambda^{2}\right) \int_{\Lambda^{2}}^{\infty} \frac{d s^{\prime}}{\pi} \frac{\phi\left(s^{\prime}\right)-\phi\left(\Lambda^{2}\right)}{\left(s^{\prime}-s-i \delta\right)\left(s^{\prime}-\Lambda^{2}\right)}\right]}
$$

where $\phi(s)=\operatorname{Im} \eta(s)$.

A very simple model consistent with positivity, the threshold behavior and a constant asymptotic limit for the phase is given by

$$
\phi(s)-\phi\left(\Lambda^{2}\right)=\pi \epsilon\left(1-\frac{\Lambda^{2}}{s}\right)^{p}
$$

with $\epsilon$ positive and $p=\left(\alpha\left(\Lambda^{2}\right)+1 / 2\right)$, as required by analyticity of the trajectory. Actually the phase $\phi(s)$ is zero at threshold. (In the above, for simplicity we are considering equal masses for the two scattering particles).

For arbitrary p, the integral in Eq.(10) is rather messy and shall be treated in detail elsewhere. For illustration, we shall explicitly exhibit the example of $p=1 / 2$, which is of interest for an S- wave resonance near threshold. For this case, the integrals in Eq.(10) can be done explicitly for the three different regions:

- Region I: $s>\Lambda^{2}$.

For $s>\Lambda^{2}, \eta_{I}$ is complex. Hence both the real and the imaginary part of the pole are different from zero.

$$
\underset{\operatorname{Re} \alpha_{I}(s)}{\operatorname{Im} \alpha_{I}(s)}=\left[\frac{s+s_{0}}{\Lambda^{2}+s_{0}}\right] \alpha\left(\Lambda^{2}\right) e^{\operatorname{Re} \eta_{I}(s)} \times \begin{gathered}
\cos \operatorname{Im} \eta_{I}(s) \\
\sin \operatorname{Im} \eta_{I}(s)
\end{gathered}
$$

where $\eta_{I}$ is given by

$$
\operatorname{Re} \eta_{I}(s)=-\epsilon \sqrt{1-\frac{\Lambda^{2}}{s}} \ln \left[\frac{1+\sqrt{1-\frac{\Lambda^{2}}{s}}}{1-\sqrt{1-\frac{\Lambda^{2}}{s}}}\right]
$$

and

$$
\operatorname{Im} \eta_{I}(s)=\pi \epsilon \sqrt{1-\frac{\Lambda^{2}}{s}}
$$


- Region II: $0<s<\Lambda^{2}$.

In region II, the trajectory is entirely real since $\operatorname{Im} \eta_{I I}=0$. It is given by

$$
\alpha_{I I}(s)=\left[\frac{s+s_{0}}{\Lambda^{2}+s_{0}}\right] \alpha\left(\Lambda^{2}\right) e^{\operatorname{Re} \eta_{I I}(s)}
$$

where

$$
\eta_{I I}(s)=-2 \epsilon \sqrt{\frac{\Lambda^{2}}{s}-1} \tan ^{-1} \frac{1}{\sqrt{\frac{\Lambda^{2}}{s}-1}}
$$

- Region III: $s<0$.

As in case II, the trajectory is entirely real with the real phase $\eta_{I I I}$ given by

$$
\eta_{I I I}(s)=-\epsilon \sqrt{1-\frac{\Lambda^{2}}{s}} \ln \left[\frac{\sqrt{1-\frac{\Lambda^{2}}{s}}+1}{\sqrt{1-\frac{\Lambda^{2}}{s}}-1}\right]
$$

Boundary conditions are satisfied and $\eta_{I I}(0)=\eta_{I I I}(0)=-2 \epsilon$.

The model exhibits two main features. First, as our initial conditions required, the pole function has a positive imaginary part vanishing at threshold. Secondly, the slopes for the space and time-like regions do differ once the trajectories acquire an imaginary part. In fact, as confirmed by experimental data in $\pi N$ charge exchange, the slopes are larger in the space-like region.

\section{Imaginary Part}

Above threshold (region I for which $s>\Lambda^{2}$ ), the formula for the imaginary part may more conveniently be written as follows

$$
\operatorname{Im} \alpha(s)=\tan \left(\pi \epsilon\left[1-\frac{\Lambda^{2}}{s}\right]^{p}\right) \operatorname{Re} \alpha(s)
$$

For high mass time-like limit $\left(\Lambda^{2} / s \rightarrow 0\right)$, Eq.(18) leads to a very simple expression

$$
\operatorname{Im} \alpha(s)=\pi \epsilon \operatorname{Re} \alpha(s)
$$

According to the previous Eq.(19), the imaginary part of the pole would be essentially linear with the squared energy just as the real part. We verify this behavior for various meson and baryon resonances. 
In Figure 1, we analyze experimental data for the $\Delta$ resonances. On the left side we show the real part of the trajectory $\left(J\right.$ vs $\left.M^{2}\right)$ through resonance masses. On the right side we show the corresponding imaginary part (as given by Eq.(8)) employing the total widths of the $\Delta$ resonances, versus the squared mass values of the $\Delta$ resonances. As we can see, the asymptotic limit described in Eq.(19) holds for this system quite accurately. The slope of Re $\alpha$ is found to be around $0.88 \mathrm{GeV}^{-2}$ and the slope of $\operatorname{Im} \alpha$ is found to be around $0.17 \mathrm{GeV}^{-2}$ confirming that the $\epsilon$ parameter in Eq.(19) is indeed quite small (about a few percent).
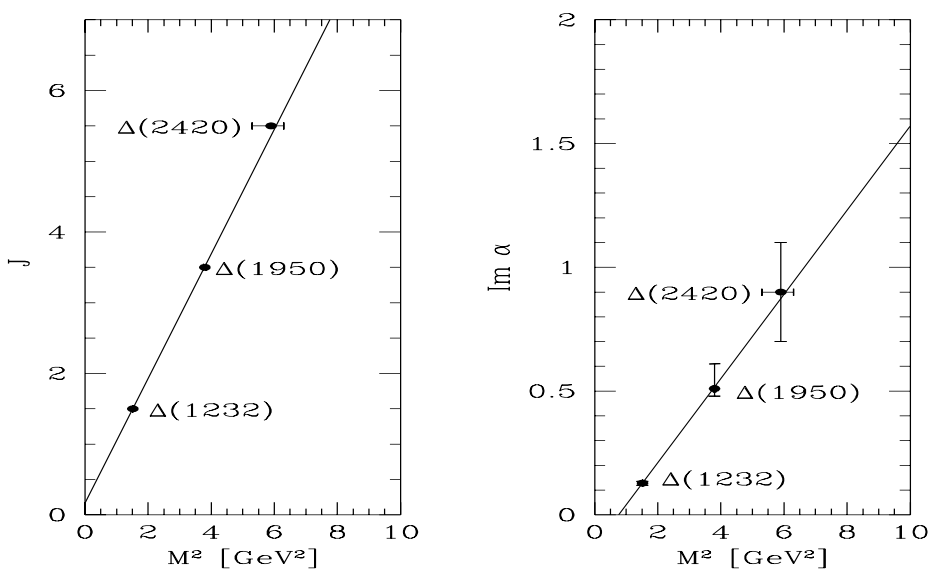

Fig. 1. The real [LEFT] and imaginary [RIGHT] part of the Regge trajectories for $\Delta$ resonances. The imaginary part of the pole is related to total widths of the hadronic states through Eq.(8).

Earlier Törnqvist, [2], had found a similar systematics for the total widths of light mesons purely phenomenologically. Wherever our Regge analysis overlaps with his, we are in complete agreement.

\subsection{Threshold Correction Factors}

In order to be able to use all resonance data lying on a given trajectory, including those for which threshold corrections are substantial, it is convenient to use the following expression for the total width,

$$
\Gamma=\left(\frac{J}{\alpha^{\prime} M}\right) \tan \left[\pi \epsilon \rho\left(M, \Lambda_{ \pm}\right)\right]
$$

where

$$
\rho=\sqrt{\left[1-\frac{\Lambda_{+}^{2}}{M^{2}}\right]\left[1-\frac{\Lambda_{-}^{2}}{M^{2}}\right]}
$$


and $\Lambda_{ \pm}=\left(m_{1} \pm m_{2}\right)$. We note that

- for a low mass resonance, such as the $\rho$, the above expression differs from its asymptotic limit by a factor two;

- for the $\omega$ resonance the width is quite small since its primary decay via the $\rho \pi$ channel occurs only through the lower tail of the $\rho$. An effective mass for $\Lambda_{+}=755 \mathrm{MeV}$ has been used;

- For $\phi(1020)$, the effective threshold factor has been determined through its P- wave decay (85\%) into $K \bar{K}$ and its S- wave decay (15\%) into $\rho \pi$ channel;

- Similarly, for $\Lambda(1520)$, the effective threshold has been determined through its decay $50 \%$ each into $N K$ (P-wave) and $\Sigma \pi$ (S- wave).

In Figure (2), we show a plot of $\Gamma_{T O T} / M \rho_{\text {eff }}$ for light flavoured mesonic systems (the $\rho, \omega, \phi$ and $K^{*}$ recurrences) and baryonic systems (the $N, \Delta$, $\Lambda$ and $\Sigma$ recurrences). Since the input total widths ranged over a factor of a hundred, it is fair to conclude that much order has been restored for each system and moreover a not too dissimilar asymptotic value for all the light systems can be inferred.

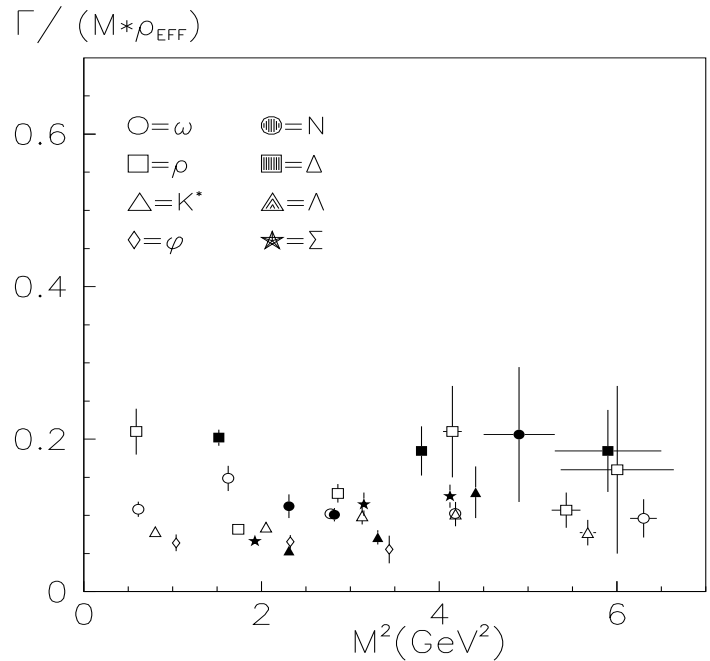

Fig. 2. $\Gamma /\left(M \rho_{E F F}\right)$ as a function of $M^{2}$ for light hadrons. $\Gamma$ is the total width, $\mathrm{M}$ the corresponding mass and $\rho$ is given by Eq.(21). $\rho_{E F F}$, as discussed in the text, has been used for $\phi(1020)$ and $\Lambda(1520)$.

\section{Slopes for Space- and Time-like Regions}

As stated earlier, slopes for the space and time-like regions of the real part of the trajectory differ once the trajectories acquire an imaginary part. The slope is seen to be larger in the space-like region, according to Eqs.(12),(13),(14) and $(17)$. 
The most suitable example to check the different values for the slope is given by the $\rho$ trajectory, where analyses of experimental data have been performed in both regions. The time-like slope is found to be around $0.88-0.9 \mathrm{GeV}^{-2},[3]$. To infer the space-like slope, we turn to the high-energy limit of $\pi^{-} p \rightarrow \pi^{0} n$. Here the $\rho$ trajectory exchange ought to be a good approximation being the only one with the right t-channel quantum numbers. Earlier analyses of the differential cross section for the above scattering had revealed that the effective $\rho$ trajectory for negative $t$ acquires a curvature and if linearity is imposed, one is led to a larger value for the slope near $\mathrm{s}=0$ : $(0.97 \pm 0.04) \mathrm{GeV}^{-2}$ in [4], $(1.08 \pm 0.03) \mathrm{GeV}^{-2}$ in [5] and (1.00 \pm 0.11$) \mathrm{GeV}^{-2}$ in [6].

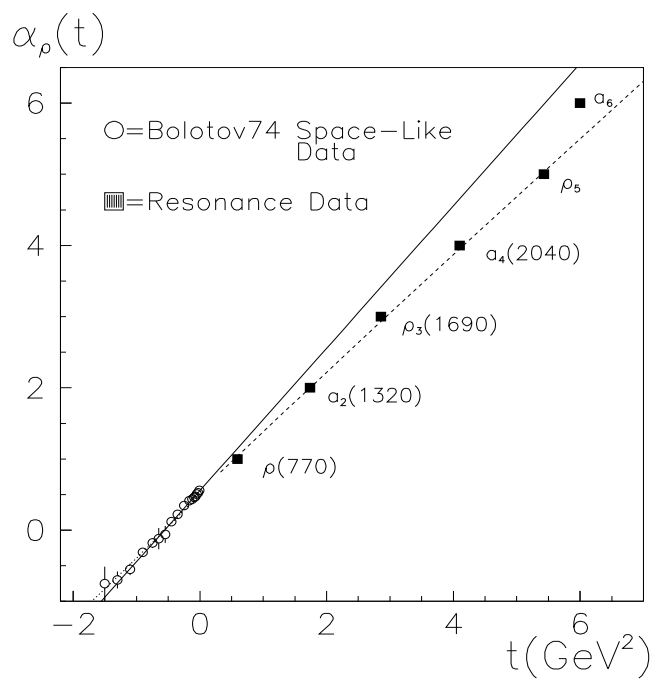

Fig. 3. $\rho$ trajectory in the space and time-like regions. Full $(\epsilon=0)$ and dashed $(\epsilon=0.03)$ lines are predictions from Eqs.(12),(13),(14) and (17). A comparison with experimental data is given. For negative $t$ values data are from the analysis in [4].

Our model describes the above picture quite well. In Figure (3) experimental data for the masses of the $\rho$ resonances and the analyses in [4] for the space-like $\rho$ trajectory are plotted again with predictions from our model, Eqs.(12),(13),(14) and (17). The full line is for $\epsilon=0$, which would imply no variation for the slopes between the two regions. The dotted and dashed lines, which are for $\epsilon=0.03$, describe data in both regions. The threshold factor which appears in Eq.(12), was taken to be $\Lambda^{2}=4 m_{\pi}^{2}$; and $s_{0}=0.56 \mathrm{GeV}^{-2}$.

For the above parameters, the intercept $\alpha_{\rho}(0)$ is found to be 0.525 , which is in good accord with the effective intercept value $\alpha_{\text {eff }}(0)=0.547$ obtained through the total cross section analysis by Donnachie and Landshoff, [7]. 


\section{Conclusions}

In this work we have shown how constraints due to analyticity and unitarity applied to a Regge pole function lead to rather powerful predictions for the hadronic systems. An admittedly simple model for the phase consistent with positivity, the threshold and asymptotic conditions was presented which appears to work reasonably well.

Since the imaginary part of the pole is directly related to the total widths of the hadronic resonance states, quantitative predictions can be made once we know the $\epsilon$ parameter, i.e. value of the asymptotic phase. For $\Delta$ and $\rho$ recurrences we found $\epsilon \approx 0.03$ to be in good agreement with data. Experimental data are consistent with our conjecture that the asymptotic phase has very nearly the same value for all of the light baryonic and mesonic sector. Moreover, whenever the threshold factor is close to one, the imaginary part of the trajectory appears to be approximately linear just as the real part is. A more complete analysis shall be presented elsewhere [8].

Regarding the real part of the trajectory, we showed that the slopes for the space and time-like regions indeed differ enough to be measurable. The analysis was presented for the $\rho$ trajectory, where much data are available, both in the

resonance region and in the space-like region through the $\pi N$ charge exchange reaction.

\section{References}

[1] Phys. Rev. D - Review of Particle Properties (1996), I-720.

[2] N.A.Törnqvist, Nucl. Phys. B(Proc. Suppl.)21 (1991), 196.

[3] N.A.Törnqvist, in Stockholm 1990, Proceedings, Low energy antiproton physics,p. 287-303 and Helsinky University preprint HU-TFT-90-52.

[4] V.N.Bolotov et al., Nucl. Phys. B73 (1974), 365; and references therein.

[5] F.Arbab and C.B.Chiu, Phys. Rev. 147 (1966), 1045.

[6] G.Höhler et al., Phys. Lett. 20 (1966), 79.

[7] A. Donnachie and P.V. Landshoff, Phys. Lett. B (1992), 227.

[8] S.Filipponi, G. Pancheri and Y. Srivastava; under preparation. 